DOI: $10.15393 /$ j3.art.2020.7190

UDC 517.54

IRYNA DENEGA

\title{
ESTIMATE OF THE MAXIMUM PRODUCT OF INNER RADII OF NON-OVERLAPPING DOMAINS
}

\begin{abstract}
In this paper, the upper estimate for the maximum of the products of inner radii of mutually non-overlapping domains is obtained for any $n$-radial system of points on the complex plane at all possible values of some parameter $\gamma$. The conditions under which the structure of points is not important in the proved results are established.
\end{abstract}

Key words: inner radius of domain, non-overlapping domains, the Green function, transfinite diameter, theorem on minimizing of the area, the Cauchy inequality

\section{Mathematical Subject Classification: 30C75}

Let $\mathbb{N}, \mathbb{R}$ be sets of natural and real numbers, respectively, $\mathbb{C}$ be the complex plane, $\overline{\mathbb{C}}=\mathbb{C} \bigcup\{\infty\}$ be its one-point compactification, $\mathbb{U}$ be the open unit disk in $\mathbb{C}$, and $\mathbb{R}^{+}=(0, \infty)$. Let $r(B, a)$ be an inner radius of the domain $B \subset \overline{\mathbb{C}}$ relative to a point $a \in B$ [1] - [9]. The inner radius of the domain $B$ is connected to Green's generalized function $g_{B}(z, a)$ of the domain $B$ by the relations

$$
\begin{gathered}
g_{B}(z, a)=-\ln |z-a|+\ln r(B, a)+o(1), \quad z \rightarrow a, \\
g_{B}(z, \infty)=\ln |z|+\ln r(B, \infty)+o(1), \quad z \rightarrow \infty .
\end{gathered}
$$

A system of points $A_{n}:=\left\{a_{k} \in \mathbb{C}, k=\overline{1, n}\right\}, n \in \mathbb{N}, n \geqslant 2$, is called $n$-radial if $\left|a_{k}\right| \in \mathbb{R}^{+}$for $k=\overline{1, n}$ and

$$
0=\arg a_{1}<\arg a_{2}<\ldots<\arg a_{n}<2 \pi .
$$

Consider the following extremal problem.

(C) Petrozavodsk State University, 2020 
The problem. For any value of the parameter $\gamma \in \mathbb{R}^{+}$, find an estimate of the maximum of the functional

$$
J_{n}(\gamma)=\left[r\left(B_{0}, 0\right) r\left(B_{\infty}, \infty\right)\right]^{\gamma} \prod_{k=1}^{n} r\left(B_{k}, a_{k}\right),
$$

where $n \in \mathbb{N}, n \geqslant 2, a_{0}=0, A_{n}=\left\{a_{k}\right\}_{k=1}^{n} \in \mathbb{C} /\{0, \infty\}$ be any $n$-radial system of different points, $B_{0}, B_{\infty},\left\{B_{k}\right\}_{k=1}^{n}$ be a system of mutually nonoverlapping domains, $0 \in B_{0} \subset \overline{\mathbb{C}}, \infty \in B_{\infty} \subset \overline{\mathbb{C}}, a_{k} \in B_{k} \subset \overline{\mathbb{C}}, k=\overline{1, n}$.

The functional $J_{n}(\gamma)$ was considered, for example, in the papers [1-4], [9], in which the following inequality for $J_{n}(\gamma)$ was established in particular cases for some values of $\gamma$ :

$$
J_{n}(\gamma) \leqslant J_{n}^{0}(\gamma):=\left(\frac{4}{n}\right)^{n} \frac{\left(\frac{4 \gamma}{n^{2}}\right)^{\frac{2 \gamma}{n}}}{\left|1-\frac{4 \gamma}{n^{2}}\right|^{\frac{2 \gamma}{n}+\frac{n}{2}}}\left|\frac{n-2 \sqrt{\gamma}}{n+2 \sqrt{\gamma}}\right|^{2 \sqrt{\gamma}} .
$$

Equality in this inequality is achieved when $0, \infty, a_{k}$ and $B_{0}, B_{\infty}, B_{k}$, $k=\overline{1, n}$, are, respectively, poles and circular domains of the quadratic differential

$$
Q(w) d w^{2}=-\frac{\gamma w^{2 n}+\left(n^{2}-2 \gamma\right) w^{n}+\gamma}{w^{2}\left(w^{n}-1\right)^{2}} d w^{2} .
$$

The following proposition is true.

Theorem 1. Let $n \in \mathbb{N}, n \geqslant 2, \gamma \in \mathbb{R}^{+}$. Then, for any fixed n-radial system of different points $A_{n}=\left\{a_{k}\right\}_{k=1}^{n} \in \mathbb{C} /\{0, \infty\}$ and any mutually non-overlapping domains $B_{0}, B_{\infty}, B_{k}, a_{0}=0 \in B_{0} \subset \overline{\mathbb{C}}, \infty \in B_{\infty} \subset \overline{\mathbb{C}}$, $a_{k} \in B_{k} \subset \overline{\mathbb{C}}, k=\overline{1, n}$, the following inequality holds:

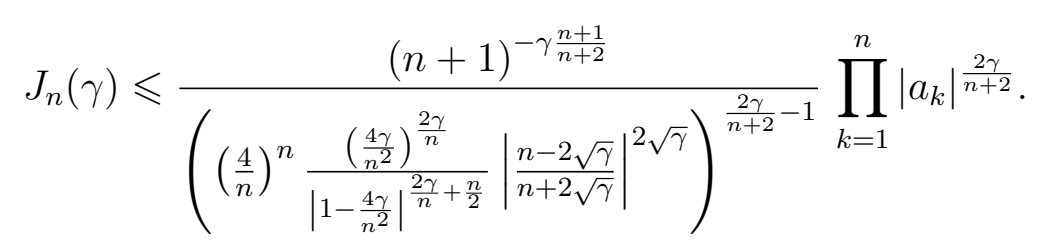

Proof. Let $J_{n}^{0}(\gamma)$ be the maximum of the functional $J_{n}(\gamma)$. In papers [1-4], [9] the authors reviewed the case when $J_{n}(\gamma) \leqslant J_{n}^{0}(\gamma)$. Consider the case $J_{n}^{0}(\gamma)=J_{n}(\gamma)$. Let $d(E)$ be the transfinite diameter of a compact set $E \subset \mathbb{C}$. Then the following relation holds

$$
r\left(B_{0}, 0\right)=r\left(B_{0}^{+}, \infty\right)=\frac{1}{d\left(\overline{\mathbb{C}} \backslash B_{0}^{+}\right)} \leqslant \frac{1}{d\left(\bigcup_{k=1}^{n+1} \bar{B}_{k}^{+}\right)},
$$


where $B^{+}=\left\{z: \frac{1}{z} \in B\right\}$. Using the well-known Polya theorem [5, p. 28], the inequality

$$
\mu E \leqslant \pi d^{2}(E)
$$

where $\mu E$ denotes the Lebesgue measure of a compact set $E$, is valid. Whence, we get

$$
d(E) \geqslant\left(\frac{1}{\pi} \mu E\right)^{\frac{1}{2}}
$$

Then, from (3) we have

$$
r\left(B_{0}, 0\right) \leqslant \frac{1}{d\left(\bigcup_{k=1}^{n+1} \bar{B}_{k}^{+}\right)} \leqslant\left(\frac{1}{\pi} \sum_{k=1}^{n+1} \mu \bar{B}_{k}^{+}\right)^{-\frac{1}{2}} .
$$

From the theorem of minimization of areas [6, p. 34] we obtain:

$$
\mu(B) \geqslant \pi r^{2}(B, a)
$$

Inequality (4) implies directly that

$$
r\left(B_{0}, 0\right) \leqslant\left(r^{2}\left(B_{\infty}, \infty\right)+\sum_{k=1}^{n} r^{2}\left(B_{k}^{+}, a_{k}^{+}\right)\right)^{-\frac{1}{2}}
$$

From the equality

$$
r\left(B_{k}^{+}, a_{k}^{+}\right)=\frac{r\left(B_{k}, a_{k}\right)}{\left|a_{k}\right|^{2}}
$$

we get

$$
r\left(B_{0}, 0\right) \leqslant\left[r^{2}\left(B_{\infty}, \infty\right)+\sum_{k=1}^{n} \frac{r^{2}\left(B_{k}, a_{k}\right)}{\left|a_{k}\right|^{4}}\right]^{-\frac{1}{2}} .
$$

In a similar way,

$$
r\left(B_{\infty}, \infty\right) \leqslant\left[r^{2}\left(B_{0}, 0\right)+\sum_{k=1}^{n} r^{2}\left(B_{k}, a_{k}\right)\right]^{-\frac{1}{2}} .
$$

Taking into account the Cauchy inequality, 


$$
\begin{gathered}
\left(r^{2}\left(B_{\infty}, \infty\right)+\sum_{k=1}^{n} \frac{r^{2}\left(B_{k}, a_{k}\right)}{\left|a_{k}\right|^{4}}\right)^{\frac{1}{2}} \geqslant \\
\qquad(n+1)^{\frac{1}{2}}\left[r\left(B_{\infty}, \infty\right) \prod_{k=1}^{n} \frac{r\left(B_{k}, a_{k}\right)}{\left|a_{k}\right|^{2}}\right]^{\frac{1}{n+1}} .
\end{gathered}
$$

Then

$$
r\left(B_{0}, 0\right) \leqslant(n+1)^{-\frac{1}{2}}\left[r\left(B_{\infty}, \infty\right) \prod_{k=1}^{n} r\left(B_{k}, a_{k}\right)\right]^{-\frac{1}{n+1}} \cdot \prod_{k=1}^{n}\left|a_{k}\right|^{\frac{2}{n+1}}
$$

Analogically,

$$
r\left(B_{\infty}, \infty\right) \leqslant(n+1)^{-\frac{1}{2}}\left[r\left(B_{0}, 0\right) \prod_{k=1}^{n} r\left(B_{k}, a_{k}\right)\right]^{-\frac{1}{n+1}} .
$$

Combining two previous inequalities, we obtain

$$
r\left(B_{0}, 0\right) r\left(B_{\infty}, \infty\right) \leqslant(n+1)^{-\frac{n+1}{n+2}}\left[\prod_{k=1}^{n} r\left(B_{k}, a_{k}\right)\right]^{-\frac{2}{n+2}} \prod_{k=1}^{n}\left|a_{k}\right|^{\frac{2}{n+2}}
$$

From the above arguments it follows that

$$
\begin{aligned}
{\left[r\left(B_{0}, 0\right) r\left(B_{\infty}, \infty\right)\right]^{\gamma} } & \prod_{k=1}^{n} r\left(B_{k}, a_{k}\right) \leqslant \\
& \leqslant(n+1)^{-\gamma \frac{n+1}{n+2}}\left[\prod_{k=1}^{n} r\left(B_{k}, a_{k}\right)\right]^{1-\frac{2 \gamma}{n+2}} \prod_{k=1}^{n}\left|a_{k}\right|^{\frac{2 \gamma}{n+2}} .
\end{aligned}
$$

Our assumption yields the relation

$$
J_{n}^{0}(\gamma)=\left[r\left(B_{0}, 0\right) r\left(B_{\infty}, \infty\right)\right]^{\gamma} \prod_{k=1}^{n} r\left(B_{k}, a_{k}\right)
$$

Obviously [8],

$$
r\left(B_{0}, 0\right) r\left(B_{\infty}, \infty\right) \leqslant 1 .
$$

Hence,

$$
J_{n}^{0}(\gamma)=\prod_{k=1}^{n} r\left(B_{k}, a_{k}\right)
$$

Therefore, we conclude that

$$
J_{n}(\gamma) \leqslant \frac{(n+1)^{-\gamma \frac{n+1}{n+2}}}{\left(J_{n}^{0}(\gamma)\right)^{\frac{2 \gamma}{n+2}-1}} \prod_{k=1}^{n}\left|a_{k}\right|^{\frac{2 \gamma}{n+2}}
$$


Thus, Theorem 1 is proved.

Remark 1. If $\gamma=\frac{n+2}{2}$ and $\prod_{k=1}^{n}\left|a_{k}\right| \leqslant 1$, then from Theorem 1, the following inequality holds:

$$
\left[r\left(B_{0}, 0\right) r\left(B_{\infty}, \infty\right)\right]^{\frac{n+2}{2}} \prod_{k=1}^{n} r\left(B_{k}, a_{k}\right) \leqslant(n+1)^{-\frac{n+1}{2}} .
$$

In this case, the structure of points and domains is not important.

From Theorem 1 we have the following statements.

Corollary 1. Let $n \in \mathbb{N}, n \geqslant 2, \gamma \in \mathbb{R}^{+}$. Then, for any system of different points $\left\{a_{k}\right\}_{k=1}^{n}$ of the unit circle $|z|=1$ and any mutually nonoverlapping domains $B_{0}, B_{\infty}, B_{k}, a_{0}=0 \in B_{0} \subset \overline{\mathbb{C}}, \infty \in B_{\infty} \subset \overline{\mathbb{C}}$, $a_{k} \in B_{k} \subset \overline{\mathbb{C}}, k=\overline{1, n}$, the following inequality holds:

$$
J_{n}(\gamma) \leqslant \frac{(n+1)^{-\gamma \frac{n+1}{n+2}}}{\left(\left(\frac{4}{n}\right)^{n} \frac{\left(\frac{4 \gamma}{n^{2}}\right)^{\frac{2 \gamma}{n}}}{\left|1-\frac{4 \gamma}{n^{2}}\right|^{\frac{2 \gamma}{n}+\frac{n}{2}}}\left|\frac{n-2 \sqrt{\gamma}}{n+2 \sqrt{\gamma}}\right|^{2 \sqrt{\gamma}}\right)^{\frac{2 \gamma}{n+2}-1}} .
$$

Remark 2. If $\gamma=\frac{n+2}{2}$, then from Corollary 1, the following inequality holds

$$
\left[r\left(B_{0}, 0\right) r\left(B_{\infty}, \infty\right)\right]^{\frac{n+2}{2}} \prod_{k=1}^{n} r\left(B_{k}, a_{k}\right) \leqslant(n+1)^{-\frac{n+1}{2}}
$$

Corollary 2. Let $n \in \mathbb{N}, n \geqslant 2, \gamma \in \mathbb{R}^{+}$and $B_{0} \subset \mathbb{U}$. Then, for any system of different points $\left\{a_{k}\right\}_{k=1}^{n}$ of the unit circle $|z|=1$ and any mutually non-overlapping domains $B_{k}, a_{k} \in B_{k} \subset \overline{\mathbb{C}}, k=\overline{0, n}$, and $B_{k}$, $k=\overline{1, n}$, are mirror-symmetric relative to the unit circle $|z|=1$, the inequality

$$
r^{2 \gamma}\left(B_{0}, 0\right) \prod_{k=1}^{n} r\left(B_{k}, a_{k}\right) \leqslant \frac{(n+1)^{-\gamma \frac{n+1}{n+2}}}{\left(\left(\frac{4}{n}\right)^{n} \frac{\left(\frac{4 \gamma}{n^{2}}\right)^{\frac{2 \gamma}{n}}}{\left|1-\frac{4 \gamma}{n^{2}}\right|^{\frac{2 \gamma}{n}+\frac{n}{2}}}\left|\frac{n-2 \sqrt{\gamma}}{n+2 \sqrt{\gamma}}\right|^{2 \sqrt{\gamma}}\right)^{\frac{2 \gamma}{n+2}-1}}
$$

holds. 


\section{References}

[1] Dubinin V. N. Symmetrization method in geometric function theory of complex variables. Russian Math. Surveys. 1994, vol. 1, pp. 1-79.

DOI: http://dx.doi.org/10.1070/RM1994v049n01ABEH002002.

[2] Kuzmina G. V. Problems on extremal decomposition of the riemann sphere. Notes scientific. J. Math. Sci. (N.Y.), 2003, vol. 118, no. 1, pp. 4880-4894. DOI: https://doi.org/10.1023/A:1025580802209.

[3] Bakhtin A. K., Bakhtina G. P., Zelinskii Yu. B. Topological-algebraic structures and geometric methods in complex analysis. $\mathrm{Zb}$. prats of the Inst. of Math. of NASU, 2008. (in Russian)

DOI: https://doi.org/10.13140/RG.2.1.1660.6242.

[4] Dubinin V. N. Condenser capacities and symmetrization in geometric function theory. Birkhäuser/Springer, Basel, 2014.

DOI: https://doi.org/10.1007/978-3-0348-0843-9.

[5] Polya G., Szego G. Isoperimetric inequalities in mathematical physics. M:Fizmatgiz, 1962. (in Russian)

[6] Goluzin G. M. Geometric theory of functions of a complex variable. Amer. Math. Soc. Providence, R.I., 1969.

[7] Jenkins J. Univalent functions and conformal mapping. Moscow:Publishing House of Foreign Literature, 256, 1962. (in Russian)

DOI: https://doi.org/10.1007/978-3-642-88563-1.

[8] Lavrent'ev M. A. On the theory of conformal mappings. Tr. Sci. Inst An USSR, 1934, vol. 5, pp. 159-245. (in Russian)

[9] Bakhtin A. K., Denega I. V. Sharp estimates of products of inner radii of non-overlapping domains in the complex plane. Probl. Anal. Issues Anal., 2019, vol. 8(26), no. 1, pp. 17-31.

DOI: https://doi.org/10.15393/j3.art.2019.5452.

Received October 22, 2019.

Accepted January 7, 2020.

Published online January 28, 2020.

Institute of Mathematics of the National Academy of Sciences of Ukraine Department of complex analysis and potential theory 01024 Ukraine, Kyiv-4, 3, Tereschenkivska st.

E-mail: iradenega@gmail.com 